\title{
Wie lässt sich die Versorgung in der Breite sichern?
}

\author{
Der Arztberuf befindet sich im Wandel: Gab es früher viele Einzelkämpfer, \\ geht der Trend heute hin zur Kooperation. KBV-Chef Dr. Andreas Köhler erläu- \\ terte, wo er den Arzt in 20 Jahren sieht, warum es nicht gelingen wird, Ärzte \\ mit mehr Honorar aufs Land zu locken, und mit welchen Herausforderungen \\ die KBV in Zukunft zu kämpfen haben wird.
}

— Nicht nur magere Honorarzuwächse, sondern insbesondere die grassierende Bürokratisierung macht den Ärzten ihren Beruf madig. In den letzten vier Jahren haben Anfragen von Kassen, die Atteste, Bescheinigungen usw. einfordern, um $27000 \%$ (!) zugenommen, $30 \%$ der ärztlichen Zeit muss in Büroarbeit investiert werden, erklärte KBVChef Dr. Andreas Köhler.

Deshalb drängt die KBV auch darauf, dass Computer in Praxen verstärkt zum Einsatz kommen. Bisher arbeiten erst rund $70 \%$ der Praxen mit EDV. Das soll ein Ende haben: Ab 1.1.2011 werden Vertragsärzte gezwungen sein, ihre Abrechnung online abzugeben.

\section{Einzelkämpfer leisten mehr Wochenstunden}

Auch wenn der Trend eindeutig weg von der Einzelpraxis hin zu Kooperationen geht: Die wenigen großen MVZs sind bereits pleite, sagte Dr. Köhler. Das liegt daran, dass in den großen MVZs viele angestellte Ärzte arbeiten und diese nicht bereit sind, mehr als 40 Wochenstunden zu leisten. Ansonsten liegt

\section{Der Trend geht ein- deutig weg von der Einzelpraxis hin zur Kooperation.}

die Arbeitszeit eines niedergelassenen Arztes bei rund 51,5 Stunden in der Woche. In einem MVZ arbeiten durchschnittlich 2,3 Ärzte. Das heißt: Bei den meisten Versorgungszentren handelt es sich um zwei Ärzte, die sich zusammenschließen, was eher einer Gemeinschaftspraxis gleichkommt.

\section{Ärztinnen niederlassungsunwillig?}

Dass Ärzte nicht mehr freiberuflich arbeiten wollen bzw. die Anstellung vorziehen, liegt auch an der zunehmenden Feminisierung des Arztberufes, meint Köhler. Inzwischen gibt es mehr Medizinstudentinnen als -studenten. Viele Frauen wollen sich nach Köhlers Aussage nicht selbstständig machen, zumal zum Zeitpunkt einer möglichen Niederlassung erst einmal Kinder zur Lebensplanung gehören.

Zudem favorisieren die jungen Frauen die Allgemeinmedizin oder die Gy-

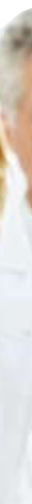

näkologie, Fächer wie Orthopädie oder Urologie sind dagegen wenig gefragt, so Köhler. Damit ist ein Mangel an Ärzten nicht nur in der Menge, sondern auch für einzelne Fachrichtungen abzusehen.

In ländlichen Gebieten ist der Ärztemangel schon zu spüren. Junge Ärzte mit mehr Geld aufs Land zu locken bringt aber nichts, sagt Köhler. Zum einen steht das Honorar bei Ärzten gar nicht an erster Stelle. Der Infrastruktur (Kindergärten, Schulen etc.) am jeweiligen Ort kommt viel mehr Gewicht zu. Zudem hat in den neuen Bundesländern bereits eine gewisse „Versteppung“ begonnen: Junge Leute wandern mangels beruflicher Möglichkeiten in die Städte $a b$, und für die ältere, sterbende Bevölkerung kommen keine "neuen“ GKV-Versicherten nach.

\section{Anschubfinanzierung ist keine Lösung} Haben die Ärzte aber nicht genügend zu versorgende Fälle auf dem Land, um ihren Lebensunterhalt auf Dauer zu sichern, nützt auch eine Anschubfinanzierung nichts. Andererseits sagen Städteplaner oder Bürgermeister immer wieder: Ohne Arzt vor Ort wird sich auch keine Industrie ansiedeln.

Bezüglich der Hausarztverträge zeigte sich Köhler naturgemäß skeptisch. Dass beide Systeme (Hausarztverband, KVen) auf Dauer nebeneinander existieren können bzw. dass die Verwaltungskosten auf dem gleichen Niveau bleiben, glaubt der KBV-Chef nicht.

ANKE THOMAS -

- Quelle: Vortrag Dr. Andreas Köhler: „Der Arztberuf im Jahr 2030“ im Rahmen einer Tagung der Metax, einem auf Ärzte spezialisierten Steuerberaterverbund, 14.5.2010, Mainz 